
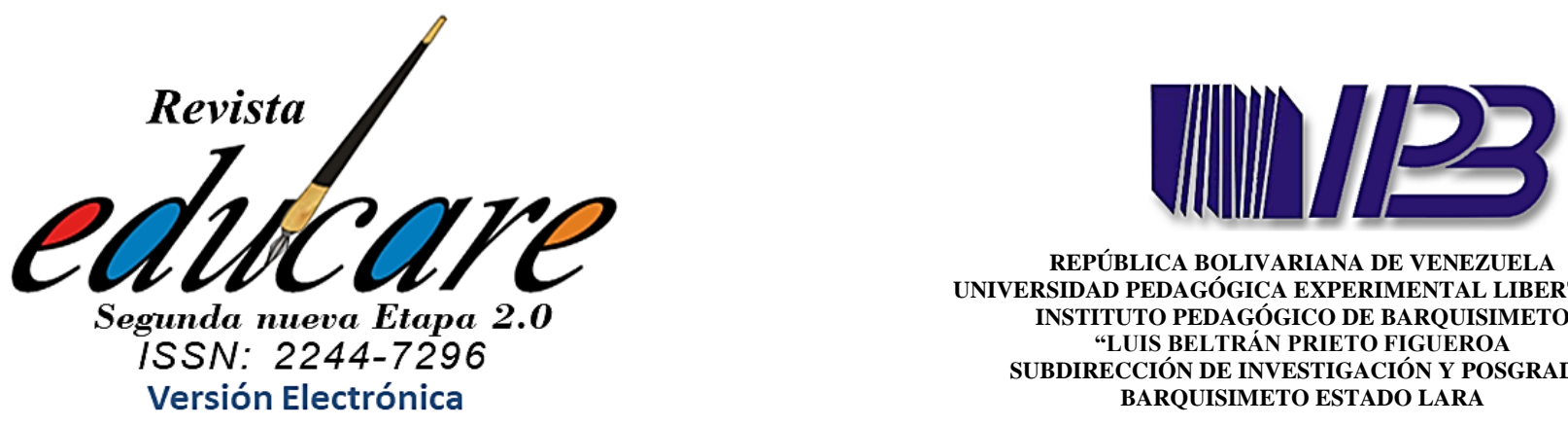

REPÚBLICA BOLIVARIANA DE VENEZUELA UNIVERSIDAD PEDAGÓGICA EXPERIMENTAL LIBERTADOR INSTITUTO PEDAGÓGICO DE BARQUISIMETO "LUIS BELTRÁN PRIETO FIGUEROA SUBDIRECCIÓN DE INVESTIGACIÓN Y POSGRADO BARQUISIMETO ESTADO LARA

Héctor Iván Guerrero Gallardo*

ORCID: https://orcid.org/0000-0002-3026-3722

Luis Enrique Noroña Casa **

ORCID: https://orcid.org/0000-0001-9297-1047

Anabel Betancourt Mejía***

ORCID: https://orcid.org/0000-0003-2917-340X

Elena Raquel Betancourt Mejía****

ORCID: https://orcid.org/0000-0003-1563-9328

UNIVERSIDAD CENTRAL DE ECUADOR

*Docente de la Universidad Central del Ecuador, Doctor PhD en Investigación Educativa - Universidad de Alicante de España, Magíster en Gerencia de Proyectos Educativos y Sociales - Universidad Central del Ecuador. Correo electrónico higuerrero@uce.edu.ec

**Docente de la Universidad Central del Ecuador Magister en Docencia Universitaria y Administración Educativa. Correo electrónico lenorona@uce.edu.ec ***Magister en docencia universitaria y administración educativa, profesora titular, Unidad Educativa San Rafael. Correo electrónico melanabel@gmail.com

****Docente de la Universidad Central del Ecuador Magister en Docencia Universitaria y Administración Educativa. Correo electrónico

\section{EL MODERADOR EN LÍNEA: UNA PROPUESTA PEDAGÓGICA PARA LA MEDIACIÓN EN LOS PROCESOS DE ENSEÑANZA Y APRENDIZAJE EN TIEMPOS DE COVID 19}

THE ONLINE MODERATOR: A PEDAGOGICAL PROPOSAL FOR MEDIATION IN TEACHINGLEARNING PROCESSES IN COVID TIMES 19 


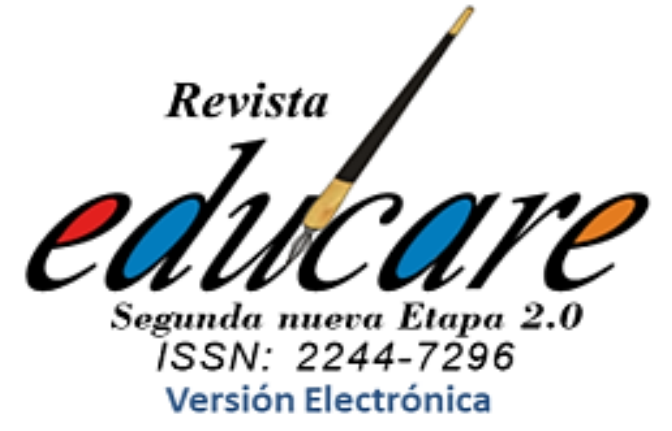

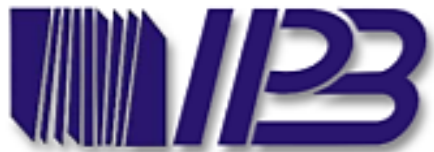

REPUBLICA BOLNARLLXA DE VENEZTELA TNTVERSIDAD PEDAGOGICA EXPERIIENTAL LIBERTADOR

INSTITUTO PEDAGÓGICO DE B.AROUTSDIETO

REPÚBLICA BOLIVARIANA DE VENEZUELA

UNIVERSIDAD PEDAGÓGICA EXPERIMENTAL LIBERTADOR

INSTITUTO PEDAGÓGICO DE BARQUISIMETO

"LUIS BELTRÁN PRIETO FIGUEROA

SUBDIRECCIÓN DE INVESTIGACIÓN Y POSGRADO BARQUISIMETO ESTADO LARA

\section{Resumen}

El presente artículo tiene como propósito presentar una propuesta pedagógica teórico práctica sobre el moderador en línea para mediar los procesos de enseñanza y aprendizaje en tiempos de Covid 19. Esta propuesta parte de una investigación documental la cual consistió en hacer un análisis crítico e interpretativo de datos secundarios, registrados por otros investigadores y fuentes oficiales en cuanto a la formación en TIC del docente ecuatoriano. Del mismo se generaron tres grandes categorías: Formación docente en TIC, Recursos TIC, y Competencias del docente en TIC; estas unidades de análisis conformaron la base de la propuesta para luego ser estructurada de la siguiente manera: Técnicas básicas para la moderación en línea, Sistemas de gestión de aprendizaje, Herramientas para comunicación sincrónica o asincrónicas y Herramientas para crear contenido. Por último a modo de reflexión se hace énfasis en la importancia de la moderación en línea y como a partir de esta nueva forma de gestionar el acto pedagógico se puede brindar una formación óptima a los niños y jóvenes del país en este tiempo histórico pandémico.

Palabras claves: moderación en línea, mediación, procesos de enseñanza y aprendizaje, Covid 19.

\section{Abstract}

THE ONLINE MODERATOR: A PEDAGOGICAL PROPOSAL FOR MEDIATION IN TEACHINGLEARNING PROCESSES IN COVID TIMES 19

The purpose of this article is to present a practical theoretical pedagogical proposal on the online moderator to mediate the teaching and learning processes in Covid 19 times. This proposal was based on a documentary research which consisted of making a critical and interpretive analysis of secondary data, recorded by other researchers and official sources regarding the TIC training of Ecuadorian teachers. Three main categories were generated from it: teaching training in TIC, TIC Resources, and TIC Teacher Skills; these units of analysis formed the basis of the proposal and then be structured as follows: Basic Techniques for Online Moderation, Learning Management Systems, Tools for Syn chronous or Asynchronous Communication, and Tools for Creating Content. Finally, as a reflection, emphasis is placed on the importance of online moderation and how from this new way of managing the pedagogical act can provide optimal training to the children and young people of the country in this pandemic historical time.

Keywords: online moderation, mediation, teaching and learning processes, Covid 19. 


\section{Introducción}

La construcción de una sociedad basada en el conocimiento tiene como eje central la educación, y en la actualidad esta debe girar en torno a nuevos paradigmas educativos y cambios pedagógicos contemporáneos los cuales apunten a la formación no sólo de competencias científicas y técnicas, sino también de competencias sociales requeridas para asumir cambios materiales, educativos y culturales medulares influenciados en este momento crucial por todo el proceso de complejidad que vive la sociedad ante la aparición del Covid 19 a nivel mundial.

Pandemia que ha obligado a los sistemas más sólidos de la sociedad a ir en búsqueda de nuevas soluciones, sustentables para dar respuesta a la ciudadanía en el orden político, educativo, social y cultural. En este sentido, las Tecnologías de la Información y la Comunicación (TIC) se han convertido como el eje articulador que mueve y conecta todos los sistemas para dar respuesta inmediata a la sociedad.

Las TIC en palabras de Ávila (2016) se convierten en la alternativa, la herramienta y el medio por excelencia para encarar con éxito los avatares de la sociedad especialmente para dar respuesta en los sistemas educativos, porque permite superar y avanzar hacia el conocimiento en una sociedad que demanda la continuidad educativa y la atención como principio para la formación y el desarrollo humano en tiempo de confinamiento.

Al respecto, García (2020) señala que el Covid-19 replanteó la manera y los métodos de enseñanza en el mundo. Hoy las aulas de clase están vacías, los estudiantes y profesores cambiaron un espacio físico por uno digital poniendo a prueba sus conocimientos, la innovación educativa y tecnológica. A partir de este hecho la educación cambió su forma de gestión y presencialidad sin perder su esencia formativa sustancial, como lo refiere la UNESCO (2019) la educación es un derecho humano para todos a lo largo de toda la vida, y el acceso a la instrucción debe ir acompañado de la calidad.

En este sentido, las TIC aplicadas a la educación se cristalizan como una ventaja tanto para los estudiantes como para el docente, pues permiten mantener la comunicación entre ellos, incluso de forma personalizada, secundado al estudiante en su propio proceso, convirtiéndolo en un agente activo en el progreso de su aprendizaje, y a los docentes en facilitadores y mediadores en la construcción y apropiación de conocimiento (Ávila, 2016). 
La UNESCO (2019) refiere que la tecnología puede facilitar el acceso universal a la educación, reducir las diferencias en el aprendizaje, apoyar el desarrollo de los docentes, mejorar la calidad y la pertinencia del aprendizaje, reforzar la integración y perfeccionar la gestión y administración de la educación.

Desde esta perspectiva se aprecia que las TIC en la educación, representan una verdadera oportunidad para el aprendizaje y el enriquecimiento cognitivo de los estudiantes, considerando que el acceso a recursos TIC, como programas y materiales puede ofrecer un entorno mucho más rico y una experiencia docente más dinámica (Ávila, 2016). Además que amplía la gama de recursos, estrategias didácticas y la modalidad de comunicación que se pueden ofrecer para el mejoramiento, optimización y mayor alcance de quehacer educativo.

De allí que el rol del docente en la labor educativa es fundamental para crear ambientes de aprendizajes que combinen el manejo del conocimiento, las metodologías participativas y el compromiso con los resultados de aprendizajes obtenidos por sus estudiantes.

Para ello es necesario asumir y desarrollar formas diferentes de enseñar y aprender, en tal sentido, el docente debe disponer de capacidades necesarias para reconocer, valorar y en su caso aceptar modelos cambiantes de aproximación al conocimiento. Las tecnologías están diseñadas para enriquecer esos nuevos espacios para la enseñanza, con nuevas normas, y novedosos modelos que a su vez precisan de nuevos profesores que deben ir ocupando estos escenarios. Con referencia a lo expresado, Godoy (2006) señala:

El docente tendría que orientar su formación profesional para aplicar la tecnología de información en los procesos de aprendizaje con el soporte que caracteriza a la sociedad de la información con la finalidad de crear condiciones y facilitar mecanismos y vías para la búsqueda del conocimiento, es decir, asumir el proceso de gestión del conocimiento. (p. 45)

Hecha esta observación, la sociedad actual y los sistemas educativos en general indistintamente del nivel solicitan la apertura de un docente mediador y moderador de experiencias de aprendizaje a través de las TIC, un docente con cualidades y condiciones distintas a los que estamos acostumbrados a ver, que esté abierto a los nuevos desafíos y considere en su formación adquirir competencias tecnológicas como herramienta clave para estar en la vanguardia de la nuevas formas de enseñar y aprender; exigencias de una nueva 
sociedad en la que el resguardo de la salud obliga el confinamiento y con ello una nueva forma de pensar en la educación.

Si bien es cierto, las TIC se encuentran a disposición de la enseñanza y del aprendizaje, en el sistema educativo tradicional la presencialidad ha sido el procedimiento estándar para llevar a cabo todo el hecho educativo. Sin embargo con la aparición del Covid 19, y los cambios emergentes se hace necesario que el docente tenga los conocimientos pertinentes de cómo llevar a cabo un proceso de moderación en línea de forma efectiva, en el cual no solo ponga a atención al cumplimiento de las tareas y objetivos programados, sino que también esté atento en la parte emocional y afectiva del estudiante.

Lo antes señalado, es un llamado a la voluntad del docente a crear comunidades de aprendizaje, en la que el estudiante protagonice de forma activa este proceso; sin que esto lo afecte emocionalmente, donde él se sienta acompañado por el docente con las orientaciones necesarias para desenvolverse en un ambiente de trabajo colaborativo.

Con base a lo referido el presente artículo tiene como propósito dar a conocer el diseño de una propuesta educativa teórica y práctica para el docente ecuatoriano con miras a mejorar la atención escolar con herramientas TIC, con una visión desde la moderación en línea en tiempo de confinamiento. Dicha propuesta es producto de una revisión documental y de estudios investigativos actuales que develan, la realidad del mencionado docente en cuanto al proceder educativo mediado por las TIC. De allí que el objetivo es orientar al docente en cuanto a ¿Cómo debe conducir la acción educativa on line? ¿Cuál es la dinámica que debe seguir? ¿Qué herramientas de gestión, comunicación y planificación pedagógicas y tecnológicas puede utilizar? ¿Con base a qué teoría educativa puede orientar la moderación? Todo ello con la finalidad de ayudar al docente ecuatoriano a encarar con éxito los desafíos del momento actual de la sociedad.

\section{El Impacto las TIC en la Educación en tiempos de Covid 19}

La situación vivida actualmente obliga a todos los sistemas educativos al replanteo profundo de la labor docente, y para ello la enseñanza no presencial demanda de recursos, habilidades y competencias docentes específicas, en vista de que el repertorio cotidiano seguido en la función escolar no cala dentro de la modalidad a distancia. 
Esto implica que es el momento de asumir grandes retos que contribuyan a una mayor eficiencia y calidad de la práctica educativa, permitiendo ver el acto pedagógico de una manera diferente e innovadora, incluso casi nunca vista, propiciando la participación, del estudiante en conjunto con la familia, desde la creatividad el pensamiento creativo e invitando el aprendizaje colaborativo, logrando de esta manera resignificar el conocimiento de forma continua.

Con base lo referido la UNESCO (2020) enfatiza lo siguiente:

En el nuevo escenario, la actividad del docente se vincula con el uso pedagógico de tecnologías digitales, la creatividad para resolver distintos retos que la situación excepcional plantea, el despliegue de estrategias de comunicación sincrónicas (inmediata) y asincrónicas (no inmediata) y el diseño de planes o guías de trabajo para desarrollar el aprendizaje autónomo, por nombrar algunos ejemplos. (p. 4)

Esto supone que al incorporar las TIC en el ámbito educativo se tiene como responsabilidad asumir cambios en diferentes aspectos, los cuales deben ir desde la formulación de los objetivos, el nuevo rol de los docentes y los estudiantes y la organización de los espacios de aprendizaje desde un contexto virtual. El docente como tal está obligado a salir de su rutina incluso asumir un nuevo rol con énfasis en la medición y la moderación como categorías centrales en el proceso de facilitar los aprendizaje. Con énfasis a lo señalado la UNESCO (2004) en el documento "Las tecnologías de la información y la comunicación en la formación docente" suscribe que con "el uso de las TIC el rol del profesor deja de ser únicamente el de transmisor de conocimiento para convertirse en un facilitador y orientador del conocimiento y en un participante del proceso de aprendizaje junto con el estudiante" (p. 43).

En tal sentido, al incorporar la TIC en este momento de confinamiento en el contexto educativo además de dar una visión diferente del docente como dador de clase le permitirá al estudiante desarrollar habilidades y poner en práctica las estrategias necesarias para administrar y evaluar el gran cumulo información que tiene a su disposición, además le atribuye la responsabilidad en conjunto con la familia de participar activamente en su formación académica, aspecto que aunque pudiese estar estipulado dentro de los reglamentos internos de las escuelas es casi inédito y crea un gran impacto que el acompañamiento de la familia sea primordial en el proceso de aprendizaje.

Otro impacto importante es que el docente se convierte en un programa autor así es 
conocido en el sistema de documentos de hipertexto (World Wide Web) como un diseñador de nuevos entornos de aprendizaje, adaptador y productor de materiales didácticos con diferentes soportes técnicos y evaluador de los procesos que se producen en estos nuevos entornos. Con referencia a lo expresado Latapí (citado por Díaz Barriga, 2008) sostiene:

Se espera que los profesores privilegien estrategias didácticas que conduzcan a sus estudiantes a la adquisición de habilidades cognitivas de alto nivel, a la interiorización razonada de valore y actitudes, a la apropiación y puesta en práctica de aprendizajes complejos, resultado de su participación activa en ambientes educativos experienciales mediados por la virtualidad y situados en contextos reales. (p. 138)

Queda claro el impacto que tiene las TIC en este momento pandémico e histórico de la humanidad, y por supuesto se revaloriza la función docente, en esta sociedad mediada por conocimiento e impulsada por las tecnologías. En tal sentido, es necesario una actitud diferente, pues la tecnología acrecienta su papel como una herramienta aliada que genera oportunidades no solo para innovar en la forma de facilitar el conocimiento, sino más bien como una estrategia que permite dar continuidad a la formación académica del estudiante y valorizar a la educación como el elemento por excelencia para conducir a los pueblos en su proceso de desarrollo.

\section{La Moderación de aprendizaje en Línea: Función del Docente}

Cuando el proceso de enseñanza se gestiona haciendo uso de las TIC (Plataforma virtuales de aprendizaje, aplicaciones web, entre otras) esa función que realiza el docente se le conoce como moderación en línea. En palabras de Bieremaet al. (2002) la moderación de aprendizaje en línea se define de la siguiente manera:

Una relación de beneficio mutuo entre el estudiante y el docente a través del ordenador,

la cual provee de aprendizaje, mejora y promueve... una relación a menudo sin límites, igualitaria y cualitativamente diferente que la tradicional educativa cara a cara. (p. 219)

En la actualidad la moderación de aprendizajes en línea se ha convertido en un gran paradigma educativo, que ha movido prácticamente el sistema educativo de toda Latinoamérica, a raíz del llamado de la Organización Mundial de la Salud (OMS) al confinamiento ante la propagación del Covid-19. 
Este proceso de gestión educativa por parte del docente se realiza a través del uso de diferentes herramientas de comunicación (Sincrónicas y Asincrónicas) la cual le permite al estudiante protagonizar activamente en su proceso de aprendizaje. Es un acompañamiento que realiza el facilitador tomando como principio estar al lado del participante, en un ambiente de trabajo colaborativo (Ávila, 2016).

En esta modalidad de enseñanza y aprendizaje, los elementos interactivos, la dimensión social y el diálogo comienzan a adquirir un gran valor. Esta interacción permite pasar de modelos basados en un aprendizaje individual donde el protagonismo académico se centra en el profesor, a un modelo de aprendizaje basado en la adquisición de conocimiento a partir de la interacción que tiene el profesor con los estudiantes y estos a su vez con diferentes materiales y herramientas de estudio.

En este sentido, Harasimet al. (2000), señalan que en la educación y formación tradicional el profesor dirige la instrucción, hace las preguntas y marca el ritmo de la clase, en cambio en la moderación en línea el aprendizaje está centrado en el participante y requiere un papel diferente del profesor, donde éste esté más cercano al estudiante y menos encargado de impartir lecciones. El énfasis de esta situación tiene que estar en el propio proceso intelectual del estudiante y en el aprendizaje colaborativo.

Por otra parte el moderador necesita contar con una serie de habilidades y cualidades que les permitan cumplir su rol, en tal sentido, que pueda preparar y presentar un programa de estudios interactivos y participativos, según Moore (2001) estas habilidades se presentan en cuatro ámbitos estrechamente relacionados: pedagógico, social, técnico y administrativo, las mismas son presentadas de la siguiente forma:

- Ámbito pedagógico: el moderador es el facilitador que contribuye con conocimientos especializados, focaliza la discusión en los puntos críticos, hace las preguntas y responde a las contribuciones de los participantes, le da coherencia a la discusión, sintetiza los puntos destacando los temas emergentes, acompaña, media y retroalimenta al estudiante en su proceso de formación, conduce el aprendizaje individual y grupal, orienta y aconseja cuando el estudiante o el grupo lo necesite.

- Ámbito social: necesita habilidades para crear una atmósfera de colaboración donde el ambiente sea agradable, acogedor, empático y que él esté siempre dispuesto a 
ayudar.

- Ámbito técnico: debe garantizar que los participantes se sienten cómodos con las herramientas web y si es necesario apoyarlos, debe poseer habilidades mínimas de carácter general relacionadas con el uso de la tecnología, la computadora y las redes.

- Ámbito administrativo: debe conocer programas y aplicaciones para poder generar conferencias, grupos de trabajo, editar información; debe utilizar las herramientas que provea el ambiente para este propósito y que permitan crear y gestionar la conferencia, hacer un seguimiento individual y grupal de la actuación de los estudiante, así como también saber administrar los equipos de trabajo.

Desde esta perspectiva se denota que la función del docente como moderador de aprendizaje en línea se entiende desde su accionar, porque desde allí él puede articular dodo un sistema de enseñanza con base a las actividades en pro al aprendizaje debidamente planificado y desarrollado con nuevas estrategias. En este sentido el docente, no puede, ni deben usar la misma dinámica o metodología que se utiliza en el marco relacional síncrono presencial, que en el asíncrono virtual.

De allí que es importante la utilización de estrategias innovadoras específicas para el desarrollo del aprendizaje en los nuevos marcos que se configuran. Hacer lo mismo de siempre usando tecnología de punta no es lo adecuado y al hacerlo se comete un gran error Medina (2014).

Sobre la base de lo anteriormente expuesto, el moderador de aprendizaje en línea debe situarse en el nuevo espacio formativo siendo guía y compañero en el proceso de formación académica del estudiante. Su rol, se fundamenta en el acompañamiento, no en ser la principal base de información o de conocimiento, en tal sentido de acuerdo a Ávila (2016), Medina (2014) y Piña (2014) sus funciones se engloban en los siguientes roles:

- Guiar desde al lado: el cual permite llevar a los participantes a un nuevo nivel conceptual o a una zona de desarrollo potencial, así mismo, capacitar a los participantes en nuevas habilidades.

- Instructor o líder de proyecto: diseñar ciclos de retroalimentación regular y manejable. 
- Líder del proceso grupal: debe liderar actividades introductorias constructoras de comunidad, de igual manera proveer de "consuelo" virtual a los tecnológicamente limitados, considerar la diversidad de intereses y antecedentes de los participantes, infundir personalidad mediante el tono, gráficos y humor, mantener y cultivar el ritmo de respuestas, sostener el ritmo que se ha marcado, organizar las aportaciones y secuencias de las discusiones y balancear la comunicación entre correo-e y discusión.

En este mismo orden de ideas, Barbera (2001) han sintetizado las tareas del moderador en el desarrollo de la discusión en tres etapas: planificación, intervención en el desarrollo de discusión, y cierre. En cuanto a la etapa de planificación los autores en referencia las especifican de la siguiente manera:

- Planificar la discusión según objetivos del debate.

- Agrupar virtualmente a los participantes.

- Especificar el formato discursivo, preparar las fases de la discusión, prever aspectos dificultosos del contenido.

- Presentar buenas preguntas o texto inicial, y prepararse para ir profundizando.

- Preparar, para proponer inicialmente, pautas para la participación (lenguaje usado, calidad-longitud, la manera de referirse a otros mensajes, el tipo de fase, el tipo de mensaje en cada fase)

- Confeccionar los puntos del contenido a tratar en la discusión.

Con relación a la intervención y desarrollo de la discusión se detallan los siguientes:

- Explicar las expectativas y objetivos de la discusión.

- Iniciar de manera significativa la discusión con preguntas o tema relevante.

- Reformular la pregunta inicial cuando las intervenciones van en dirección equivocada.

- Organizar la discusión.

- Coordinar las participaciones y participar ofreciendo contenido específico no sólo mensajes de gestión del debate.

- Favorecer un mayor grado de reflexión y profundidad en las participaciones. 
- Favorecer el centrar el tema de la discusión (resúmenes, relación con contenidos del curso, dar alternativas para tratar otros temas).

- Ofrecer retroalimentación y realizar recapitulaciones si el debate es extenso.

- Registrar información de cada estudiante para recordar su conocimiento, experiencias e interés.

- Finalizar la discusión o líneas de discusión cuando ésta se prolongue sin producir resultados con relación a la construcción de conocimiento.

De igual manera, para el cierre de la discusión se puntualiza lo siguiente:

- Ofrecer un resumen articulado de las intervenciones.

- Cerrar la discusión de manera explícita.

- Valorar las intervenciones públicamente y, si corresponde personalmente en privado.

- Relacionar la temática de la discusión con acciones educativas posteriores (artículos, direcciones Web, listas de interés, grupos de discusión, etc). vinculados con el tema.

A la luz de las consideraciones anteriores, existen una serie de herramientas de comunicación las cuales son factores claves en la moderación en línea. Las mismas según Holmberg (1989, citado por Padilla et al., 2015) son conversaciones didácticas guiadas, que permiten la interacción y el diálogo, elementos centrales en la calidad de la educación a distancia. Asimismo al presentarse una efectiva comunicación se está evitando la ampliación de una brecha comunicacional entre profesores y estudiantes y una interrupción en el desarrollo y progreso en el aprendizaje.

Las funciones y/o roles del docente como moderador en línea varían de acuerdo al autor, no obstante cada uno de su desempeño es valioso para darle efectividad, crédito, valía a esta acción docente. 


\section{Tabla 1}

Roles del moderador en línea desde la mirada de diferentes autores

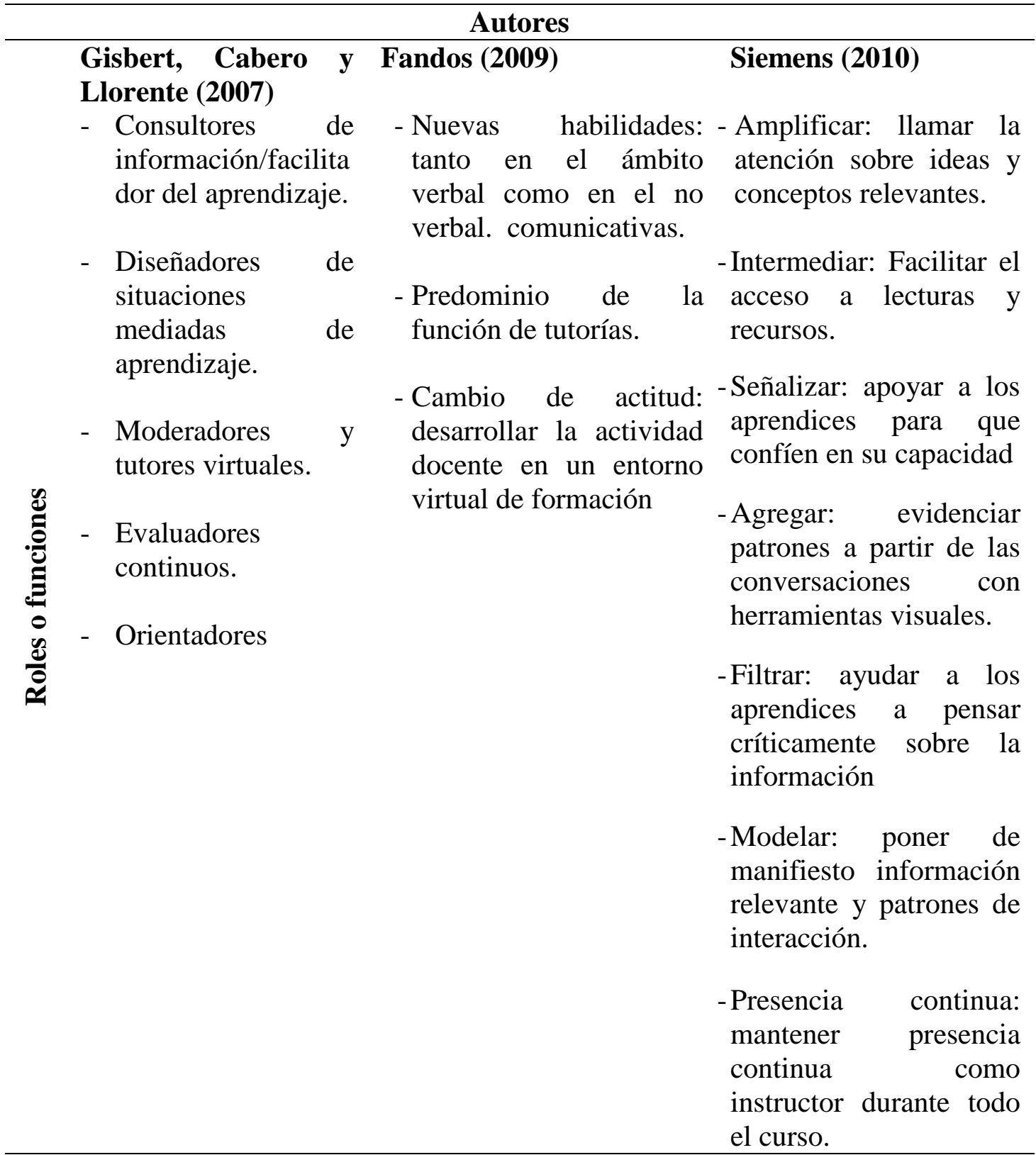

Nota: Tomado de Padilla et al. (2015), adaptación propia.

Los canales de comunicación para la continuidad pedagógica y moderación en línea en tiempos de COVID-19

En el mejor de los escenarios, hace referencia el docente se encuentra ante el desafío de enseñar a la distancia en una comunidad de estudiantes con acceso a una 
computadora/ordenador con conectividad a internet. La diversidad de realidades en que se desarrolla el proceso de enseñanza-aprendizaje a distancia dificulta proponer una estrategia de comunicación estandarizada. La selección de los canales de comunicación dependerá de posibles indicaciones o acuerdos promovidos por el equipo directivo, de los recursos tecnológicos que tengan los estudiantes en los hogares (dispositivos y conectividad a internet), de la etapa educativa y de las competencias digitales docentes y del estudiante. Más allá de las particularidades de cada docente (Rappoport, et al., 2020)

En este sentido es importante que existan acuerdos no solo a nivel de las escuelas sino también de estas con la familia para compartir recursos, materiales, conocimientos, experiencias lo cual es fundamental para hacer más eficiente el trabajo docente, pero, además, son necesarios para proponer procesos de aprendizaje a distancia viables.

En este acuerdo, es competencia del docente realizar un inventario de aquellos recursos que le son más permisibles para la continuidad pedagógica y por ende poder realizar una moderación más pertinente. Entre ellos debe destacar aquellos recursos para la gestión del conocimiento, recurso para comunicación tanto sincrónica como asincrónica, recurso para la creación de contenidos.

\section{Mediación de los procesos de Enseñanza y aprendizaje}

El uso de las (TIC) con fines educativos promete, según Palloff y Pratt (citado por Borges, 2007) abrir nuevas dimensiones y posibilidades en los procesos de enseñanzaaprendizaje, entre las que cabe mencionar las siguientes:

(a) Ofertan una gran cantidad de información interconectada para que el usuario la manipule, (b) Permiten una mayor individualización y flexibilización del proceso instructivo adecuándolo a las necesidades particulares de cada usuario, y (c) Representan y transmiten la información a través de múltiples formas expresivas provocando la motivación del usuario y ayudan a superar las limitaciones temporales y/o distancias geográficas entre docentes y educandos, de este modo facilitan extender la formación más allá de las formas tradicionales de la enseñanza presencial. 
Con base a lo anteriormente referido, se destaca que todo conocimiento que se adquiere de manera intencionada, mediante un proceso diseñado para ello, implica una mediación en el tratamiento de la información, las estrategias y los medios que promueven el aprendizaje. De allí que el valor formativo (pedagógico) de las TIC está en su potencialidad mediadora porque posibilita promover, desencadenar y acompañar el aprendizaje.

En palabras de Schneuwly et al. (2012) haciendo uso del pensamiento vigostkyano señala que la mediación como proceso para lograr el avance del desarrollo, actúa como un apoyo interponiéndose entre el individuo y su entorno para ayudarle a organizar y a desarrollar su sistema de pensamiento. Los mediadores son instrumentos que transforman la realidad en lugar de imitarla. Su función no es adaptarse pasivamente a las condiciones del medio, sino modificarlas activamente (Schneuwly et al., 2012).

A la luz de lo referido, se entiende que el concepto de mediación se encuentra vinculado al pensamiento y supone una actividad humana para transformar la naturaleza como consecuencia de la reacción natural del ser humano sobre la misma. Vygotsky (1981, citado por Lima, 2005) señala que mediación actúa en la interacción humano-ambiente, ya que usa signos para hacerse entender, los cuales han sido creados por las sociedades a lo largo de la historia, en donde la comunicación es una acción mediadora del hombre.

Los procesos formativos en general y los sustentados en las TIC en particular, se establecen en ámbitos de relación, de nexo, de unión, conexión o contacto entre los elementos personales que configuran el proceso (docente-estudiante, estudiante entre sí) y entre éstos y los elementos no personales (objetivo, contenido, métodos, formas, medios, etc.). Pero a su vez el proceso necesita de nexos específicos que medien entre el enseñar y el aprender y entre estos y la realidad a aprender (Lima, 2005).

Desde esta perspectiva, el aprendizaje sustentado en TIC es mediado y en tal sentido es considerado un proceso comunicacional, en tanto se emite un mensaje que se ha hecho de una selección de aspectos representativos de la realidad, conforme a un punto de vista particular o de los fines del proceso educativo. La inserción de las TIC a la enseñanza y el aprendizaje, señala Lima (2005) se hacen con el propósito de mediar. Solo así los recursos tecnológicos constituyen un medio y no una finalidad. Un medio que contribuye en el marco del modelo pedagógico de los nuevos ambientes de aprendizaje a optimizar la actividad y la 
comunicación de los maestros con los alumnos, de estos entre sí, y de ellos con el contenido de enseñar.

\section{Teorías en que se sustenta la Moderación en línea de aprendizajes}

El proceso de moderación de aprendizaje en línea tiene sustentación teórica, y claramente se refleja en la teoría sociocultural de Lev Vigostky el cual hace énfasis en que el aprendizaje es un fenómeno social en el que los estudiantes adquieren los elementos necesarios para apropiarse del conocimiento, a través de la interacción con sus pares, profesores y el material. Además Vigostky (1978) destaca el concepto de zona de desarrollo próximo:

La distancia entre el nivel de desarrollo, dado por la capacidad de resolver independientemente un problema y el nivel de desarrollo potencial, determinado a través de la resolución de un problema bajo la guía de un adulto o en colaboración con otro compañero más capaz. (p. 133)

Y es esto precisamente lo que sucede con la moderación en línea, se crea un ambiente social de interacción a través del trabajo colaborativo, los mismos permiten transitar desde modelos de aprendizaje basados en la transmisión de conocimiento, a modelos basados en la construcción de conocimiento. De esta forma, los participantes se vuelven agentes activos en el proceso de aprendizaje.

Es necesario tener en cuenta que, si bien los entornos virtuales de aprendizaje, favorecen el aprendizaje colaborativo y la construcción de conocimiento a través de la interacción sociocultural, el conocimiento se adquiere en forma personal, pero para ello debe existir un elemento muy importante; el mismo está centrado en la actuación del moderador el cual debe ejercer una serie de roles específicos que conlleven con éxito al proceso de enseñanza y aprendizaje; donde los participantes pasen de una actuación pasiva, a una actuación activa, de una zona de desarrollo real a una zona potencial.

\section{Marco Metodológico}

La naturaleza de la investigación se orienta en el paradigma positivista, también denominado empirista; así lo destaca Muñoz y Pérez (2002), quienes lo definen como un 
"modelo de interpretación científica que establece como criterios básicos de análisis lo cuantificable y medible" (p. 245). En consecuencia, la metodología correspondiente es la cuantitativa, descrita por estos autores "como aquella en la que se recogen y analizan datos numéricos sobre variables" (p. 245).

\section{Tipo de Investigación}

El tipo de investigación es documental, la misma según Bernal (2006) consiste en un análisis de la información escrita sobre un determinado tema, según (Arias (2012), define: la investigación documental es un proceso basado en la búsqueda, recuperación, análisis, crítica e interpretación de datos secundarios, es decir, los obtenidos y registrados por otros investigadores en fuentes documentales: impresas, audiovisuales o electrónicas. Como en toda investigación, el propósito de este diseño es el aporte de nuevos conocimientos (p. 27) en este caso la moderación en línea de aprendizaje en tiempo de Covid 19. En este mismo sentido para Hernández et al. (2006) "la investigación documental depende fundamentalmente de la información que se obtiene o se consulta en documentos, se entiende este como todo material al que se puede acudir como fuente referencial sin que se altere su naturaleza o sentido" (p. 110). Para esta investigación se hizo la consulta de fuentes tanto primaria como secundaria relacionadas en primer lugar con el tema (libros digitales, revistas indexadas, documentos legales).

\section{Diseño de la Investigación}

El diseño empleado en la investigación es el descriptivo, secuencial documental, este diseño según Hurtado (2008) permite describir un evento según el tiempo, y dado que "el investigador no puede tener acceso a la fuente viva obtiene los daos a través de la revisión de documentos (p. 237).

\section{Técnica de Investigación}

La técnica utilizada fue la revisión documental, la misma según Hurtado (2008) "se recurre a la información escrita ya sea bajo la forma de todos que pueden haber sido producto de medicines hechas por otro o como textos que en sí mismo constituyen los eventos de estudio" (p. 427), de allí que para efecto de este estudio se realizó la revisión de trabajos de investigación recientes, noticias en periódico de la zona, documentos legales del Ministerio de 
Educación. Las categorías de análisis utilizadas en la revisión documental fueron las siguientes:

\section{Figura 1}

Categorías de análisis

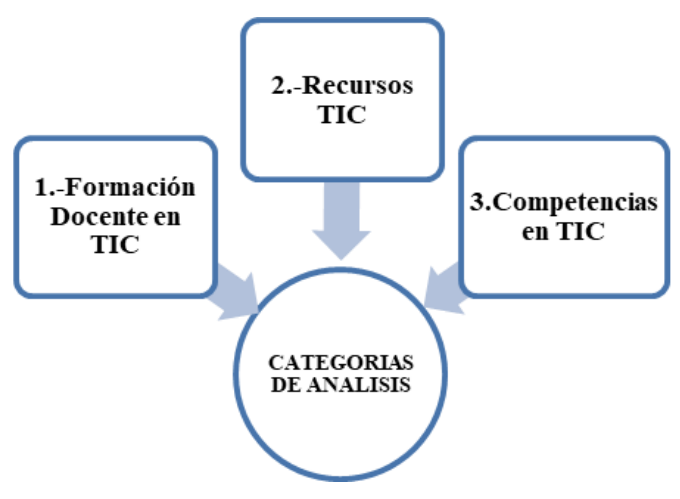

Nota: Elaboración propia.

\section{Formación de docentes en el uso de las TIC}

En a la formación para los docentes en TIC, el ministerio de Educación ha hecho conjuntamente con la empresa privada un plan de acción, el mismo forma parte de una agenda que prepara al país para la cultura digital. Ese proceso de formación ha tenido como objetivo ayudar al docente en el manejo del computador y de otras herramientas TIC.

\section{Figura 2}

Docentes formados en TIC

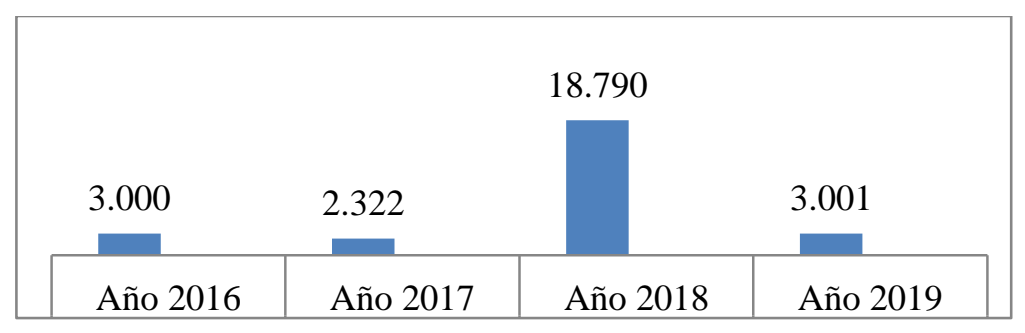

Nota: Tomado de Navarrete, G., Mendieta, R. (2018).

Como se puede apreciar en la gráfica este proceso de formación ha tenido aceptación por los docentes; sin embargo el contenido de los talleres está más dirigido con el conocer es decir con los contenidos conceptuales en cuanto el uso del computador y el manejo de herramientas TIC, dejando a un lado el hacer lo cual concierne con los contenido procedimentales y actitudinales, este último aspecto es muy importante al momento de asumir el rol de moderador en línea para mediar los aprendizajes. 
A luz de lo referido Molina (2004, citado por Tolozano et al., 2016) señala que es de suma importancia la actitud del docente al asumir la atención educativa en línea y para ello es necesario que en su perfil profesional poseer los siguientes atributos:

- Preparación sólida en el área disciplinar.

- Motivación para desempeñarse en la actividad.

- Capacidad creativa para resolver dudas o problemas.

- Actitudes positivas para guiar y orientar el aprendizaje independiente.

- Estar actualizado en las áreas de tecnología y didáctica.

- Capacidad para promover el trabajo cooperativo de sus estudiantes.

- Capacidad para identificar dificultades de los alumnos en adquisición de aprendizajes.

- Capacidad para presentar situaciones didácticas que permitan la vinculación del conocimiento al contexto de la vida real.

En este mismo sentido se puede apreciar, los objetivos del programa de formación de Tecnología de la Información y Comunicación aplicada a la Educación el cual es un programa de formación continua del Magisterio Fiscal de Ecuador, en el mismo se visualiza que ninguno de los objetos está dirigido en cómo hacer la moderación.

\section{Tabla 2}

Objetivos de formación de las TIC aplicadas a la Educación

\begin{tabular}{|c|c|c|}
\hline $\begin{array}{l}\text { Adquirir conocimientos en el } \\
\text { uso de recursos tecnológicos } \\
\text { que permitan utilizar y } \\
\text { desarrollar páginas web, blogs, } \\
\text { foros, presentaciones de } \\
\text { diapositivas, chat, wikis, etc., } \\
\text { para su adecuada aplicación } \\
\text { dentro del aula. }\end{array}$ & $\begin{array}{l}\text { Adquirir conocimientos sobre } \\
\text { las aplicaciones educativas que } \\
\text { ofrecen la web y otras que se } \\
\text { encuentren gratuitamente y saber } \\
\text { cómo utilizarlas en el aula. }\end{array}$ & $\begin{array}{l}\text { Conocer sobre los } \\
\text { motores de búsqueda } \\
\text { existentes para realizar } \\
\text { investigaciones o dirigir } \\
\text { a sus estudiantes en } \\
\text { investigaciones en } \\
\text { Internet. }\end{array}$ \\
\hline $\begin{array}{l}\text { Reconocer las herramientas } \\
\text { necesarias para buscar en } \\
\text { Internet y evaluar los recursos } \\
\text { didácticos más adecuados para } \\
\text { el trabajo del docente }\end{array}$ & $\begin{array}{l}\text { Preparar actividades centradas } \\
\text { en el contenido, par que al } \\
\text { estudiante pueda: introducir, } \\
\text { practicar, profundizar la } \\
\text { investigación, crear- desarrollar- } \\
\text { producir, usar referencias y } \\
\text { repasar (treasure hunt, webtasks, } \\
\text { Web Quests, quizzes, etc.). }\end{array}$ & $\begin{array}{l}\text { Aplicar los } \\
\text { conocimientos } \\
\text { adquiridos en proyectos } \\
\text { de clase }\end{array}$ \\
\hline
\end{tabular}

Nota: Basado en Ministerio de Educación (2012) 


\section{Recursos TIC utilizados por las docentes}

Los recursos Tic utilizados del programa de formación de Tecnología de la Información y Comunicación aplicada a la Educación como se puede apreciar en el cuadro está centrado en la búsqueda de información y algunos recursos para crear, que si bien es cierto son importante que el docente los conozca y además los tenga el conocimiento en el uso. Sin embargo existe otro tipo de recurso que le puede ser más útil al momento de asumir la atención en línea como moderador de aprendizaje.

Tabla 3

Recursos Tic

\begin{tabular}{l|lllll}
\hline \multicolumn{5}{c}{ Recurso /Descripción } \\
\hline Google & & & & & \\
\end{tabular}

Nota: Basado en Ministerio de Educación (2012). Elaboración propia

Con referencia a lo señalado, Cacheiro (2011) señala que los recursos utilizados por el docente para mediar el aprendizaje a través de las TIC, deben provocar encuentros o situaciones, desarrollar habilidades cognitivas, apoyar sus estrategias metodológicas, o facilitar o enriquecer la evaluación. Para ello la autora propone una tipología en tres categorías: información, colaboración y aprendizaje.

Los recursos TIC para la información permiten obtener datos e informaciones complementarias para abordar una temática forma actualizada en fuentes de información y formatos multimedia. Algunos recursos TIC informativos son: webgrafía, enciclopedias virtuales, bases de datos online, herramientas web 2.0 (Marcadores sociales, YouTube, Slideshare, ...), buscadores Visuales, etc.

Los recursos TIC para la colaboración permiten participar en redes de profesionales, instituciones, entre otras. El trabajo colaborativo permite llevar a cabo una reflexión sobre los 
recursos existentes y su uso en distintos contextos,entre los recursos TIC de colaboración se pueden mencionar las listas de distribución, los grupos colaborativos, herramientas web 2.0 como las wiki y los blog, Webinar, otros.

Los recursos TIC para el aprendizaje posibilitan el llevar a cabo los procesos de adquisición de conocimientos, procedimientos y actitudes. Las TIC como recursos de aprendizaje permiten pasar de un uso informativo y colaborativo a un uso didáctico para lograr los resultados de aprendizaje. Algunos recursos de aprendizaje basados en TIC son: repositorios de recursos educativos, tutoriales interactivos, cuestionarios online, herramientas web 2.0 (eBooks, Podcast, etc.) y los cursos online en abierto.

\section{Competencias TIC}

Con referencia a lo antes señalado, Tolozano et al. (2016) en una investigación realizada sobre las competencias en las TIC del docente ecuatoriano determino que necesitan formación para desarrollar la competencia digital; asimismo entre los hallazgos encontraron que los docentes tienen cierto dominio sobre aspectos técnicos, mas no utilizan la tecnología para la práctica docente pues ésta requiere habilidades o capacidades de mayor nivel. El uso pedagógico de herramientas web 2.0 es prácticamente nulo.

Por su parte, Valencia et al. (2016) sostienen que el objetivo de un plan de formación fundamentado en la apropiación de las TIC debe partir del desarrollo de una serie de competencias en el uso educativo de ellas. En este caso, se privilegian las competencias relacionadas con el diseño, la implementación y la evaluación de espacios educativos significativos mediados por TIC, las cuales se refieren a las habilidades de planificación y organización de elementos que permitan la construcción de escenarios educativos apoyados en TIC para el aprendizaje significativo y la formación integral del estudiante.

Las competencias relacionadas con la implementación dan cuenta de las habilidades que permiten poner en marcha el diseño y planificación de un escenario educativo, y que se ven reflejadas en las prácticas educativas de un docente. Finalmente, las competencias de evaluación se relacionan con las habilidades que le permiten al docente valorar la efectividad para favorecer el aprendizaje significativo en los estudiantes al incorporar las TIC a sus prácticas educativas. 


\section{Presentación de la Propuesta}

La siguiente propuesta pedagógica está dirigida a los (as) docentes del Sistema Educativo Ecuatoriano, la misma tiene doble connotación: Teórica y Práctica, dado que en primer lugar pone a disposición de los usuarios los conocimientos básicos de este proceso de moderación, y luego a modo de ejemplo y de forma didáctica deja ilustrado de cómo hacer la moderación. En este sentido el objetivo general de esta propuesta es: Brindar orientaciones pedagógicas que le permitan al docente fortalecer sus conocimientos en TIC para la moderación en línea de los aprendizajes en tiempo de Covid 19. La propuesta está organizada siguiendo la siguiente estructura:

\section{Técnicas Básicas para la moderación en línea}

Voz: La voz del moderador es una herramienta, según su aplicabilidad invita a la reflexión, a la ampliación y conlleva a la dinamización del aprendizaje.

Tabla 3

Uso de la Voz: Orientaciones para el docente

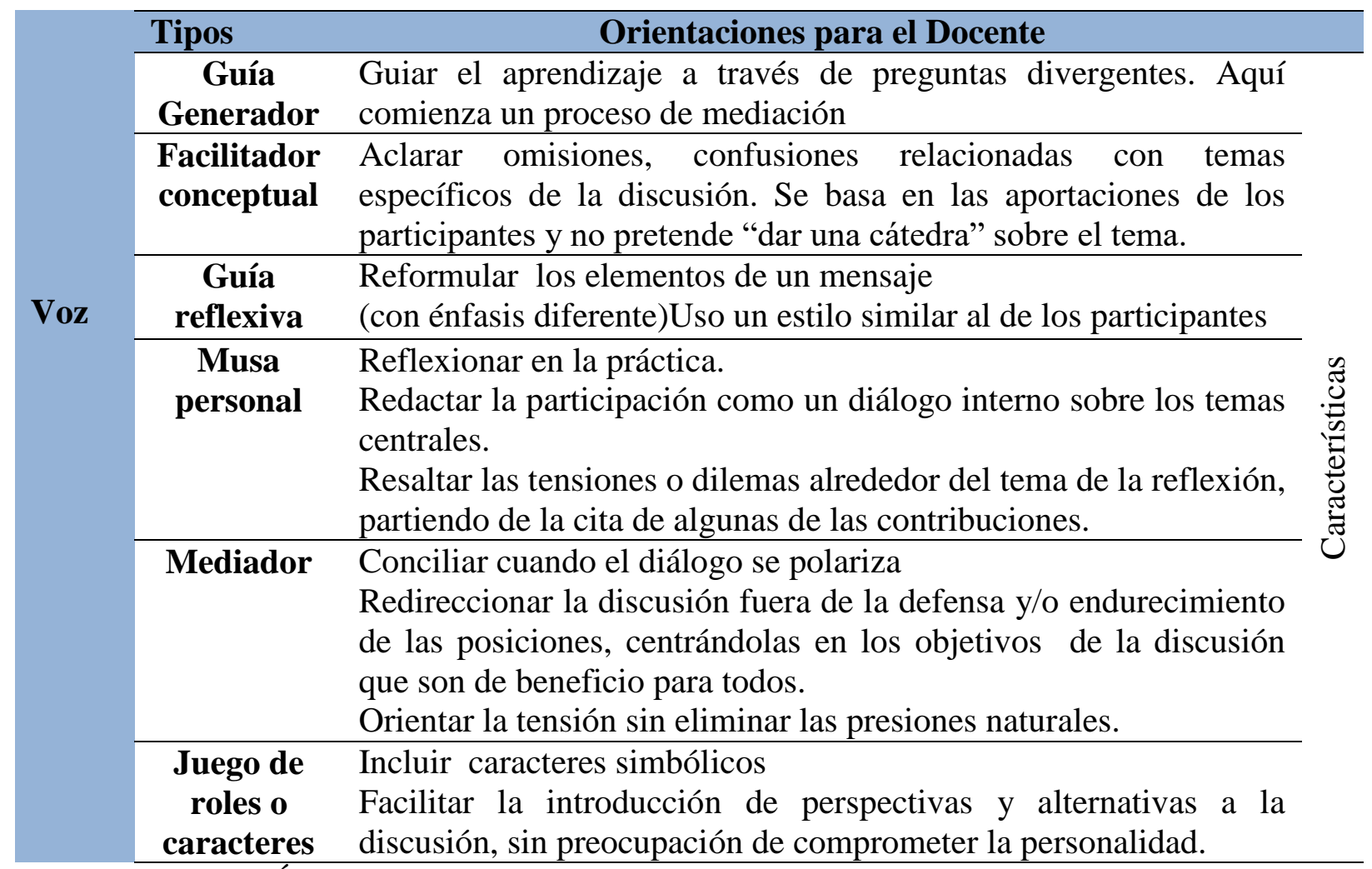

Nota: Basado en Ávila (2016) y Dávila (2016). Elaboración propia. 
Tono: Es entendido como la forma que debe utilizar el moderador para expresarse de manera amistosa, invitadora, abierta y amable. El tono en el moderador es importante para que el estudiante sienta a su docente ante todo como un ser humano y no como una máquina que le da instrucciones.

\section{Tabla 4}

Uso del Tono: orientaciones para el docente



Nota: Basado en Ávila (2016) y Dávila (2016). Elaboración propia.

\section{Sistemas de Gestión de aprendizaje}

Un Sistema de Gestión de Aprendizaje (Learning Management System, LMS), es una herramienta informática, habitualmente de gran tamaño, que permite la gestión y presentación de materiales educativos a estudiantes. El objetivo de estas herramientas es permitir el aprendizaje en cualquier parte y en cualquier momento. La mayoría de estas herramientas son herramientas web, es decir, herramientas que se usan a través de Internet utilizando un navegador web (Fernández et al, 2016).

Los entornos de gestión de aprendizaje son de gran ayuda para estar en contacto con los estudiantes y sus familias y para optimizar el tiempo de trabajo docente. 


\section{Figura 4}

Entornos de gestión
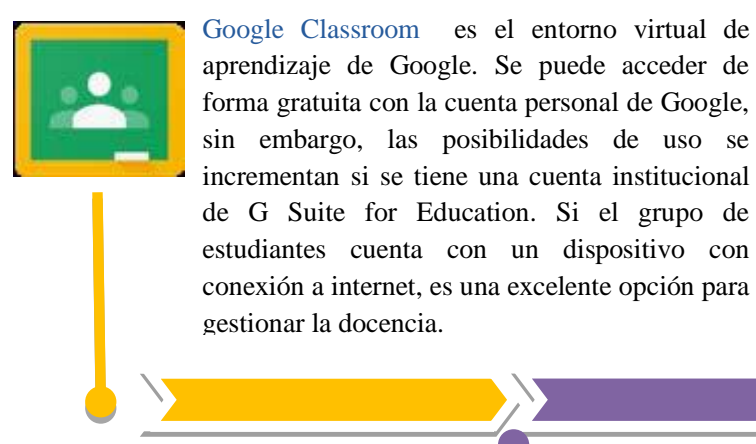

Nota: Tomado de Rappoport, et. al., UNESCO (2020).

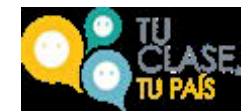

Comunidad TU CLASE es una herramienta para crear espacios ilimitados de colaboración y trabajo en línea, ya sean abiertos (públicos) o cerrados (por invitación). Permite compartir con otros docentes, familiares o estudiantes documentos, calendario, listas de enlaces, listas de tareas, reflexiones, imágenes y videos, y trabajar en wikis colaborativas

\section{Herramientas Para Crear Contenido Sistemas de Gestión de aprendizaje Técnicas Básicas para la moderación en línea}

Los videos y los encuentros virtuales son excelentes recursos para desarrollar un contenido, generar encuentros, resolver dudas y mantener vínculos de cercanía. Las siguientes alternativas son relativamente sencillas de utilizar.

\section{Figura 5}

Herramientas de creación de contenido

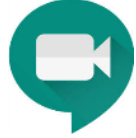

\section{Google Meet}

Permite sumar a 100 personas de forma Se puede compartir un enlace para que los estudiantes se unan a la reunión

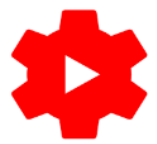

\section{YouTube Studio}

Permite grabar y subir videos de forma muy sencilla. Los videos disponen de un espacio de comentarios que puede ser útil para responder dudas de los estudiantes.

\section{Zoom}

Es una aplicación que permite realizar videollamadas con grupos de hasta 100 personas.

Las reuniones duran 40 minutos (versión gratuita). El docente puede enviar un enlace para que los estudiantes accedan a la reunión.

Nota: Tomado de Rappoport, et. al., UNESCO (2020). Adaptación propia. 


\section{4.- Herramientas Para comunicación Sincrónica o Asincrónicas Herramientas Para Crear Contenido Sistemas de Gestión de aprendizaje Técnicas Básicas para la moderación en línea}

\section{Figura 6}

\section{Herramientas de comunicación}



Whatsapp es la aplicación para teléfonos de mayor popularidad. Permite intercambiar mensajes de texto, audios, imágenes, archivos, videos y realizar videollamadas (solo 4 personas). Se pueden armar grupos con muchos usuarios y listas de difusión. Se recomienda utilizar para comunicaciones más informales, breves e inmediatas, como resolver dudas sencillas, transmitir ánimo y saludar.

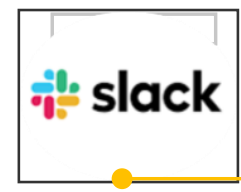

Slack es un sistema de mensajería que permite crear temas de conversaciones para gestionar los intercambios entre estudiantes y docentes de forma ordenada. También permite adjuntar documentos, realizar llamados y videollamadas. Se recomienda usar para generar mayor interacción entre las personas del grupo de clase.

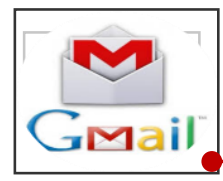

Gmail es el servicio de correo electrónico de Google. Su uso es muy extendido y poseeuna herramienta de chat y videollamada (Hangouts). Se sugiere su uso en el caso de no contar o tener dificultades técnicas con correo electrónico institucional.

Nota: Tomado de Rappoport, et. al., UNESCO (2020). Adaptación propia.

\section{Figura 7}

\section{Herramientas de no conectividad}



El mensaje de texto (SMS) Este canal de comunicación permite al docente mantener un vínculo asiduo con la familia y el estudiante de forma relativamente personalizada y ágil. Su uso se recomienda para compartir información, sugerir actividades cortas, brindar recomendaciones y orientaciones concretas, así como para mantener el vínculo y contacto personalizado.

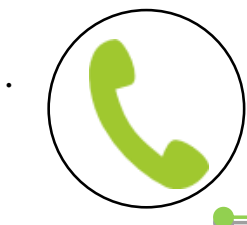

El Teléfono Es una excelente opción de acercamiento personalizado que permite al docente sostener el vínculo con cada uno de los niños, niñas o jóvenes y con sus familias

Nota: Tomado de Rappoport, et. al., UNESCO (2020). Adaptación propia. 


\section{Plan de moderación}

\section{Tabla 5}

Ejemplo de plan de moderación

\begin{tabular}{|c|c|c|}
\hline RECURSO & FUNCIÓN & $\begin{array}{l}\text { FRECUENCIA DE LA } \\
\text { MODERACIÓN }\end{array}$ \\
\hline 2 & $\begin{array}{l}\text { Organizar la clase, estimular el tiempo para } \\
\text { el entrega, Utilizar, aquí puede utilizar } \\
\text { cualquier tipo voz, puede hacerle } \\
\text { seguimiento a los estudiante }\end{array}$ & $\begin{array}{l}\text { Diaria emplear de } 1 \text { a } 2 \\
\text { horas, depende del grupo } \\
\text { de estudiante }\end{array}$ \\
\hline & $\begin{array}{l}\text { De acuerdo a la disponibilidad del } \\
\text { estudiante puede hacer conexión sincrónica } \\
\text { y brindar explicación en vivo, como también } \\
\text { Puede gravar todas las clases, y utilizar } \\
\text { diferentes tipo de tono, además de recursos } \\
\text { adiciones como powerpoint }\end{array}$ & $\begin{array}{l}\text { A principio y al final de } \\
\text { la semana }\end{array}$ \\
\hline - @. & $\begin{array}{l}\text { Orientar sobre el trabajo, aclarar dudas, } \\
\text { animar al estudiantes }\end{array}$ & $\begin{array}{l}\text { Una vez a la semana y de } \\
\text { acuerdo a las demandas de } \\
\text { los estudiantes }\end{array}$ \\
\hline slack & $\begin{array}{l}\text { Por aquí puede crear grupo entre } \\
\text { estudiantes y animarlos a realizar trabajos } \\
\text { colaborativos }\end{array}$ & $\begin{array}{l}\text { Puede crear u tema por } \\
\text { semana }\end{array}$ \\
\hline & $\begin{array}{l}\text { Mantener vínculo con los estudiantes y la } \\
\text { familia, también sirve para aclarar dudas }\end{array}$ & Una vez cada 15 días \\
\hline
\end{tabular}

Nota: Tomado de Rappoport, et. al., UNESCO (2020). Adaptación propia.

Es recomendable elaborar un protocolo donde se describen los canales de comunicación disponibles, plazos de entrega de material, frecuencias de intercambios, horarios de conexión sincrónica y cualquier otra información de relevancia que permita clarificar a los estudiantes (o familias) el sistema de comunicación diseñado. Disponer de un protocolo de comunicación, además, permitirá organizar mejor los tiempos de trabajo docente y no abrumar a las familias y estudiantes con una comunicación excesiva. 


\section{Reflexiones Finales}

Sin duda alguna este periodo pandémico que vive la sociedad divide la historia del siglo XXI, y con ella la educación de una totalmente presencial a una totalmente virtual. Si bien es cierto la escuelas, los docentes y la familia son sorprendidos de forma inaudita por este fenómeno, pues nadie estaba preparado para el momento, de allí que la tecnología con toda sus bondades se convierte y la alternativa y la respuesta para encarar la situación de la mano del docente.

Sin embargo las TIC por sí sola no suplen al docente y este a su vez necesita empoderarse de todo el conocimiento para poder asumir y manejar con éxito este momento histórico en el cual toda sociedad le demanda. Moderar en línea el aprendizaje es un proceso que requiere no solo de la dimensión pedagógica del docente es decir todo sus conocimiento, sino también de su dimensión personal, porque aunque no tenga cara a cara a los estudiantes la táctica como persona es importante para que el estudiante se sienta en compañía.

Para lograr lo anteriormente expuesto, es preciso que el moderador utilice cualidades mediadoras tales como: las voces, los tonos y las estrategias de pensamiento crítico, herramientas que según su aplicabilidad invita a la reflexión, a la ampliación y conlleva a la dinamización del aprendizaje.

Lo importante en todo este proceso es la actitud que debe asumir el docente y por sobre todo debe creer en sus potencialidades, para incursionar en esta nueva forma de enseñar y aprender, tener un visión diferente de su accionar pedagógico y de las TIC le permitirá avanzar y con él el estudiante en el aprendizaje. Desde una perspectiva general se puede concretar que la moderación en línea es una habilidad que cuenta con principios, roles y estrategias esenciales en donde su foco primordial es la formación del estudiante, por lo tanto es una labor que puede ser aprendida.

\section{Referencias}

Arias, F. (2006). El proyecto de Investigación. Introducción a la metodología científica. Caracas, Editorial Episteme. 
Avila (2016). Comunidad virtual como herramienta de apoyo al proceso de enseñanza aprendizaje. [Investigación realizada para optar a la categoría agregado]. Universidad Pedagógica Experimental Libertador Instituto Pedagógico "Luis Beltrán Prieto Figueroa"

Baltasar Fernández, B. Moreno, P. Sierra, J. y Martínez, I. 2016). Uso de las tic en educación: $\quad$ contexto $\quad y \quad$ participantes. http://ares.cnice.mec.es/informes/16/contenido/2.htm\#up

Barbera J. (2001) La educación a distancia en transición. Nuevas tendencias y retos. México, Universidad de Guadalajara. http://biblioteca.udgvirtual.udg.mx/jspui/bitstream/1234567/1873/1/Educaci\%C3\%B3n \%20a\%20distancia\%20\%A9xico.pdf

Bernal, C. (2006). Metodología de la Investigación. México, D.F., Pearson educación.

Bierema, L. y Merriam S. (2002). E-mentoring: Using Computer Mediated Communication to Enhance the Mentoring Process. Innovative Higher Education, 26 (3). http://icareermanagement.com/e-mentoring.pdf.

Borges Sáiz, F., 2007. El estudiante de entornos virtuales. Una primera aproximación. Digithum, (9). http://doi.org/10.7238/d.v0i9.520

Cacheiro, M. (2011). Recursos educativos tic de información, colaboración y aprendizaje Píxel-Bit. Revista de Medios y Educación, (39), 69-81. http://espacio.uned.es/fez/eserv.php?pid=bibliuned:425-Mlcacheiro-

\section{0\&dsID=Documento.pdf}

Dávila, O. (2016). Técnicas básicas de Moderación de Cursos en línea. http://mriuc.bc.uc.edu.ve/bitstream/handle/1ISBN-9789801290162.pdf?sequence=1

Diaz, Barriga F. (2008). Los desafíos de las TIC para el cambio educativo. Organización de Estados Iberoamericanos para la Educación, la Ciencia y la Cultura (OEI). http://www.oei.es/metas2021/LASTIC2.pdf

García, M. (2020). Educación digital en tiempos de Covid-19. https://www.forossemana.com/articulo/id/44921/nota_educacion_digital_en_tiempos_d e_covid-19 
Godoy (2006). Constructos teóricos que fundamentan las competencias del docente universitario para la gestión del conocimiento en contextos virtuales de aprendizaje. [Trabajo de Grado] Universidad de los Andes. http://www.human.ula.ve/doctoradoeneducacion/documentos/tesis_godoy.pdf

Harasim, L., Hiltz, S., Turoff, M. y Teles, L. (2000). Redes de Aprendizaje: Guía para la Enseñanza y el Eprendizaje en red, Barcelona: Gedisa/EDIUOC. http://gedisamexico.com/catalogo/educacion/557-redes-aprendizaje.html

Hernández, R; Fernández, C y Baptista, P. (2006). Metodología de la Investigación. México, D.F., Mc Graw Hill.

Hurtado, J. (2005). Cómo Formular Objetivos de Investigación. Caracas, Editorial Sypal.

Lima S. (2004). La mediación pedagógica con tecnología en la universalización de la formación de profesores. X Convención internacional "Informática2004".

Medina, E. (2014). Virtualización didáctica de la planificación instruccional. 1ra edición. Carabobo - Venezuela.

Ministerio de Educación (2012). Tecnología de la información y la comunicación aplicada a la educación. https://educacion.gob.ec/wp-content/uploads/downloads/2013/03/SiProfeTIC-aplicadas.pdf

Moore, M. (2001). La educación a distancia en los Estados Unidos: estado de la cuestión, ciclo de conferencias sobre el uso educativo de las Tecnologías de la Información y Comunicación y la educación virtual. Universidad Abierta de Cataluña, Barcelona, España. http://www.uoc.es/web/esp/art/uoc/moore/moore.html

Muñoz, A., y Pérez, C. (2002). Metodología de la Investigación. Editorial Trillas. México

Navarrete, G. Mendieta, R. (2018). Las tic y la educación ecuatoriana en tiempos de internet. Revista Espirales, 2(15) DOI: https://doi.org/10.31876/re.v2i15.220

Padilla, G., Leal, F., Hernández, M., Cabero, J. (2015). Un reto para el profesor del futuro: la tutoría virtual https://idus.us.es/bitstream/handle/11441/40462/Un_reto_para_el_profesor_del_futuro_ la_tutoria_virtual2.pdf;jsessionid=BFBE32549DF1FA492BCDF9ADA60444ED?seque $\underline{\text { nce }=1}$ 
Paulsen, A. (2002). Elementos para el análisis de la interacción educativa en los nuevos entornos de aprendizaje. Pixel-bit: revista de medios y educación, 19, 49-61. https://recyt.fecyt.es/index.php/pixel/article/view/61458

Piña, M. (2014). Moderación, por una educación presencial enriquecida. Revista de Tecnología de Información y Comunicación en Educación, 8(2). http://servicio.bc.uc.edu.ve/educacion/eduweb/v8n2/art09.pdf

Rappoport, S., Rodríguez, M., y Bresanello, M., (2020). Enseñar en tiempos de COVID-19. Publicado por la Organización de las Naciones Unidas para la Educación, la Ciencia y la Cultura. https://es.unesco.org/themes/tic-educacion

Schneuwly, B., Bronckart., J. (2012). Vigostky hoy. Editorial Popular Superiores, Barcelona: Crítica.

Tolozano, S. Lara, L. y Illescas, S. (2016). Actitudes y aptitudes del tutor para enfrentar el desafío de la formación en la modalidad dual. Revista Universidad y Sociedad, 8(1), 8191.http://scielo.sld.cu/scielo.php?script=sci_arttext\&pid=S2218$\underline{36202016000100013 \& \operatorname{lng}=\mathrm{es} \& \operatorname{tlng}=\mathrm{es} .}$

UNESCO (2004). Las tecnologías de la información y la comunicación en la formación docente. París: UNESCO: https://es.unesco.org/themes/tic-educacion

UNESCO (2019). Las TIC en la educación. https://es.unesco.org/themes/tic-educacion

Valdivieso, T. y Gonzáles, M. (2016). Competencia Digital Docente: ¿Dónde estamos? Perfil del docente de educación primaria y secundaria. El caso de Ecuador. Pixel-Bit: Revista de Medios y Educación, 49, 57-73. http://doi 10.12795/pixelbit.2016.i48.09

Valencia, T., Ochoa, S. Caicedo, A., Montes, J. y Chávez, J. (2016). Competencias y estándares TIC desde la dimensión pedagógica: Una perspectiva desde los niveles de apropiación de las TIC en la práctica educativa docente. http://www.unesco.org/new/fileadmin/MULTIMEDIA/FIELD/Santiago/pdf/Competenc ias-estandares-TIC.pdf

Vigostky, L.S. (1978). El desarrollo de los procesos psicológicos. Madrid-España. 